Check for updates

Cite this: RSC Adv., 2019, 9, 5834

\title{
Mussel-inspired nano-silver loaded layered double hydroxides embedded into a biodegradable polymer matrix for enhanced mechanical and gas barrier properties
}

\author{
Long Mao, (DD ab Jing-yi Liu, ${ }^{a}$ Si-jie Zheng, ${ }^{a}$ Hui-qing Wu, ${ }^{* c}$ Yue-jun Liu, ${ }^{\star a b}$ Zhi-han Lib \\ and Yong-kang Bai $\mathbb{B D}^{d}$
}

In this paper, a facile, green and mussel-inspired method is presented to prepare silver loaded layered double hydroxides (Ag-LDHs@PDA and Ag-LDHs@TA-Fe(III)) using a pre-synthesis polydopamine (PDA)/ tannic acid (TA)-Fe(III) layer as a nanoscale guide and PDA/TA itself as a reducing reagent to form uniform silver nanoparticles (AgNPs) on the surface of modified LDHs. Meanwhile, another kind of LDH, $\mathrm{Ag}-\mathrm{LDHs}(\mathrm{PVP})$, was prepared via the direct reduction of the precursor $\left[\mathrm{Ag}\left(\mathrm{NH}_{3}\right)_{2}\right]^{+}$with polyvinyl

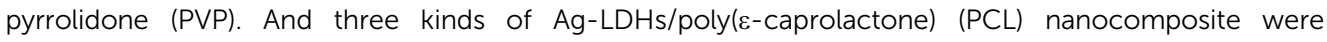
prepared by blending $\mathrm{Ag}-\mathrm{LDHs}$ and pure $\mathrm{PCL}$ via a solution casting method to obtain homogeneous films. It is shown that the obtained AgNPs are distributed on the LDH surfaces uniformly. And the high loading and medium size of the AgNPs present in Ag-LDHs(PVP) give it the best antibacterial properties. However, compared with Ag-LDHs(PVP), the better dispersibilities of Ag-LDHs@PDA and Ag-LDHs@TA$\mathrm{Fe}(\mathrm{III})$ contribute to the greater aspect ratios of $\mathrm{Ag}-\mathrm{LDH}$ in the matrices, resulting in an increase in the number of tortuous paths for gas diffusion. Meanwhile, Ag-LDHs@PDA and Ag-LDHs@TA-Fe(III) have stronger interactions with the PCL matrix, which is favorable for the existence of less interface defects in the matrix, resulting in an improvement in the mechanical and gas barrier properties. Therefore, musselinspired antibacterial Ag-LDHs/PCL nanocomposites show preferable mechanical and gas barrier properties.

Received 22nd November 2018 Accepted 4th February 2019

DOI: $10.1039 / \mathrm{c} 8 \mathrm{ra09602c}$

rsc.li/rsc-advances including use in nanomedicine, as catalyst supports, as functional additives, in water pollution treatment, and so on. ${ }^{3}$

In general, organic coatings are commonly applied to modify clay to improve the interfacial compatibility between clay minerals and matrices. Inspired by the versatile adhesion capabilities of marine adhesive proteins, polyphenolic compound surface modifications have been discovered as a powerful method to form adhesive and multifunctional coatings (such as polydopamine (PDA) and tannic acid (TA)$\mathrm{Fe}(\mathrm{III})$ complexes) on almost any substrate, such as metal oxides, clay and fibers. ${ }^{4,5}$ According to the literature, ${ }^{6-10} \mathrm{PDA}$ is capable of interacting strongly with polymers via hydrogen bonds and covalent bonds, hence, it can act as a bridge at organic-inorganic interfaces. Therefore, LDHs@PDA has been prepared via growing PDA in situ onto LDH surfaces. ${ }^{6,7}$ And PDA coatings can further improve the dispersibility and interfacial interactions of LDHs in polymer matrices, correspondingly improving the properties. To the best of our knowledge, the surface modification of LDHs using a TA-Fe(III) complex has been seldom reported. According to previous studies, ${ }^{11,12}$ TA can provide polydentate ligands for metal ions to form uniform TA-Fe(III) coatings rapidly due to the strong chelating ability of TA..$^{12-14}$ 
These brilliant works have inspired us to consider the possibility of employing a TA-Fe(III) complex as an effective and functional coating on the surface of LDHs. Meanwhile, compared with PDA, TA-Fe(III) is relatively cheap and readily available, making its widespread application possible. ${ }^{\mathbf{1 5}}$ Therefore, we proposed a facile, green and mussel-inspired method to prepare surface modified LDHs coated by TA-Fe(III) (LDHs@TA$\mathrm{Fe}(\mathrm{III}))$ in situ for the first time in our previous publication. ${ }^{\mathbf{1 6}}$

In recent years, nanostructured materials have been considered as promising candidates to act as novel antibacterial agents in the functional materials field, owing to their unique physical and chemical properties. Many nanostructured materials (such as $\mathrm{TiO}_{2}, \mathrm{ZnO}$, gold and $\mathrm{Ag}$ ) have been intensively investigated. ${ }^{\mathbf{1 , 1 7}, 18}$ Among these, Ag-based nanomaterials have been extensively used as effective antibacterial agents against a wide range of microorganisms (e.g., bacteria, fungi and viruses), because of the relatively low toxicity of silver to humans. Previous research has revealed that $\mathrm{Ag}$ nanoparticles (AgNPs) can directly react with proteins containing sulfur both inside and outside the cytomembranes of bacteria, causing structural changes or functional damage to the cytomembranes of bacteria and further affecting the viability of the bacterial cells. ${ }^{17,19-22}$ According to previous studies, ${ }^{23,24}$ polyphenolic compounds (e.g., PDA and TA) can be used as active templates for the synthesis of AgNPs due to their abundant catechol and amine groups, and they exhibit extraordinary active features, such as excellent adhesive and reducing abilities. To summarize, polyphenolic coatings are able to act as reductants, binding reagents and universal platforms for secondary reactions. Hence, PDA/TA-Fe(III) coatings can provide an excellent platform to bind and reduce silver precursor $\left[\mathrm{Ag}\left(\mathrm{NH}_{3}\right)_{2}\right]^{+}$ions in situ into metallic AgNPs on LDHs (named Ag-LDHs@PDA and Ag-LDHs@TA-Fe(III)), as shown in Scheme 1. To the best of our knowledge, compared with Ag-LDHs@PDA, Ag-LDHs@TA$\mathrm{Fe}(\mathrm{III})$ prepared via this method has been rarely reported. Meanwhile, for comparison, Ag-LDHs(PVP) was prepared via the direct reduction of silver precursor $\left[\mathrm{Ag}\left(\mathrm{NH}_{3}\right)_{2}\right]^{+}$ions with polyvinyl pyrrolidone (PVP). Ag-LDHs@PDA, Ag-LDHs@TA$\mathrm{Fe}(\mathrm{III})$ and Ag-LDHs(PVP) are collectively known as Ag-LDHs in this paper.

Aliphatic polyesters are recognized as some of the most promising materials to act as the main sources of environmentally friendly biodegradable plastics. ${ }^{25} \mathrm{Poly}(\varepsilon$-caprolactone) (PCL) is a linear and semicrystalline aliphatic polyester that can be slowly degraded by microorganisms. ${ }^{26}$ The excellent physical properties, biological compatibility and commercial availability of PCL make it an attractive substitute for conventional nonbiodegradable polymers, giving rise to common applications (e.g. packaging, biomedicine and agriculture). However, for practical applications of PCL, specific packaging requirements need to be satisfied (e.g. antibacterial and gas barrier properties). ${ }^{27}$ In order to satisfy these requirements, three kinds of AgLDHs were applied to modify the PCL matrix. Therefore, antibacterial Ag-LDHs/PCL nanocomposites were prepared via a solution casting method to obtain homogeneous films. The purpose of our research is to provide more selectivity for the multifunctional modification of LDHs, to improve the interface compatibility of Ag loaded LDHs, and promote the application of LDHs for aliphatic polyester use.

\section{Experimental section}

\subsection{Materials}

MgAl-LDHs was prepared via a modified urea method followed by hydrothermal treatment. ${ }^{6}$ Tannic acid (TA, analytical purity), $\mathrm{FeCl}_{3} \cdot 6 \mathrm{H}_{2} \mathrm{O}$ (analytical purity), dopamine hydrochloride (DA, purity of over 98\%), tris(hydroxymethyl)aminomethane (Tris, purity of over 99.9\%), polyvinylpyrrolidone (PVP, average molecular weight $=24000, \mathrm{~K} 23-27$ ), $\mathrm{AgNO}_{3}$ (purity of over 98\%), and $\mathrm{NH}_{3} \cdot \mathrm{H}_{2} \mathrm{O}$ (purity of over $25 \%$ ) were purchased from Shanghai Aladdin Bio-Chem Technology Co., Ltd. Commercial poly( $\varepsilon$-caprolactone) (PCL, Capa ${ }^{\mathrm{TM}} 6800, M_{\mathrm{w}} \geq 80000$ ) was supplied by Perstorp. All other reagents were of analytical purity and used without further purification.

\subsection{The preparation of Ag-LDHs}

Original LDHs (MgAl-LDHs), LDHs@PDA and LDHs@TA-Fe(III) were synthesized using the methods reported in our previous publications.6,16 Generally, LDHs@PDA (or LDHs@TA-Fe(III)) $(0.1 \mathrm{~g})$ was dispersed in ultrapure water $(10 \mathrm{~mL})$ under continuous sonication for $30 \mathrm{~min}$. Freshly prepared $\left[\mathrm{Ag}\left(\mathrm{NH}_{3}\right)_{2}\right]^{+}$ion solution (10 mL, 0.02 M) was dropwise added to the LDHs@PDA (or LDHs@TA-Fe(III)) dispersion under magnetic stirring, and the system was kept under stirring in an ice bath for $1 \mathrm{~h}$. After centrifugation and washing with ultrapure water three times, the product was dried in a freeze dryer.

$\mathrm{Ag}$-LDHs(PVP) was prepared via the direct reduction of the precursor $\left[\mathrm{Ag}\left(\mathrm{NH}_{3}\right)_{2}\right]^{+}$with PVP. Generally, original LDHs (0.1 g) were dispersed in ultrapure water $(10 \mathrm{~mL})$ under continuous sonication for $30 \mathrm{~min}$. Freshly prepared $\left[\mathrm{Ag}\left(\mathrm{NH}_{3}\right)_{2}\right]^{+}$ion solution $(10 \mathrm{~mL}, 0.02 \mathrm{M})$ was added to the LDH dispersion under magnetic stirring at room temperature for $1.5 \mathrm{~h}$. Then, PVP aqueous solution ( $50 \mathrm{~mL}, 0.5 \mathrm{mM}$ ) was added for stabilization and reduction, and the product was stirred at $70{ }^{\circ} \mathrm{C}$ for $7 \mathrm{~h}$. The product was collected via centrifugation and finally dried in a freeze dryer.

\subsection{The preparation of Ag-LDHs/PCL nanocomposites}

The mass fractions of Ag-LDHs in the Ag-LDHs/PCL nanocomposites were set to $0.5 \%, 1 \%$ and $3 \%$, respectively. AgLDHs was dispersed in dimethyl formamide under continuous sonication for $30 \mathrm{~min}$. Then commercial PCL was added to the dispersion at $40{ }^{\circ} \mathrm{C}$ under magnetic stirring for $1.5 \mathrm{~h}$ and the dispersion was heated to $80{ }^{\circ} \mathrm{C}$ under magnetic stirring for $1.5 \mathrm{~h}$. Afterwards, the dispersion was treated with continuous sonication for $15 \mathrm{~min}$. Finally, the dispersion was vaporized to obtain homogeneous films in horizontal PTFE molds at $60^{\circ} \mathrm{C}$.

\subsection{Characterization}

$\mathrm{X}$-ray diffraction (XRD) patterns were recorded with a powder diffractometer (X'pert, Panalytical), using $\mathrm{CuK} \alpha$ radiation at a scanning rate of $5^{\circ} \mathrm{min}^{-1}$ (from $10^{\circ}$ to $80^{\circ}$ ). The microstructures of the Ag-LDHs/PCL nanocomposites were measured via 


\section{Mussel-inspired functionalization of $L D H$ s for synthesizing Ag- LDHs@polyphenol coatings as antibacterial materials}

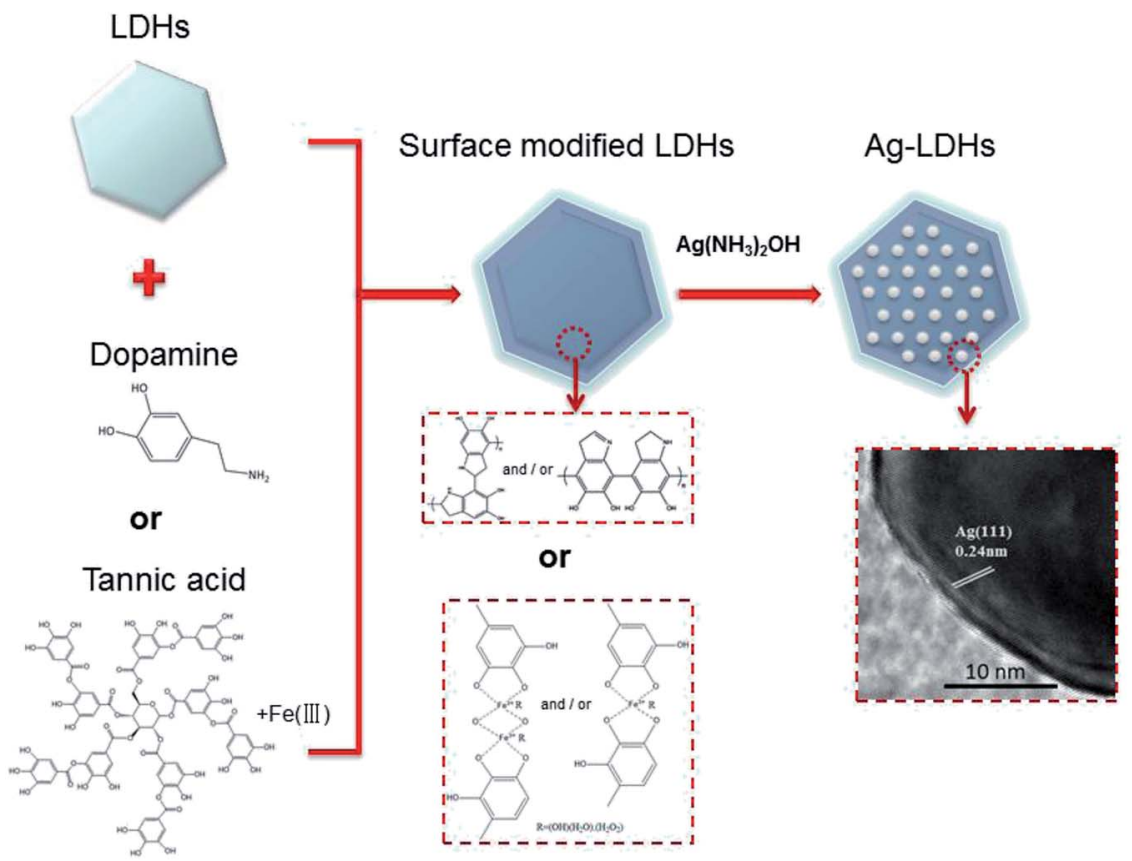

Scheme 1 A schematic illustration of the preparation of Ag-LDHs.

scanning electron microscopy (SEM, Sigma500, Zeiss). In order to maintain the original fractured surfaces of the Ag-LDHs/PCL nanocomposites, the samples were treated with liquid nitrogen to obtain fractured surfaces. The microstructure of Ag-LDHs was measured via transmission electron microscopy (TEM, Talos, FEI). And energy-dispersive X-ray spectrometry (EDS, X-Max ${ }^{\mathrm{n}}$, Oxford) measurements were taken using TEM and SEM to identify coatings around the original LDHs. UV-visible absorption spectra were recorded using a UV-visible spectrophotometer (SPECORD 210 PLUS, Analytik Jena) over the wavelength range of 300-800 $\mathrm{nm}$. Thermal properties analysis was carried out with a differential scanning calorimeter (DSC, DSC214, Netzsch). The samples were heated from room temperature to $150{ }^{\circ} \mathrm{C}$ at $20^{\circ} \mathrm{C} \mathrm{min}^{-1}$ and kept at this temperature for $5 \mathrm{~min}$ to eliminate any thermal history. Then the samples were cooled down to $-30{ }^{\circ} \mathrm{C}$ at a rate of $10^{\circ} \mathrm{C} \mathrm{min}^{-1}$ and heated to $150{ }^{\circ} \mathrm{C}$ at a rate of $10^{\circ} \mathrm{C} \min ^{-1}$. To estimate the crystallinity $(\chi)$ of the PCL matrix, the following equation was used: $\chi=\left[\Delta H_{\mathrm{m}} /\left(\Delta H_{0} \times \varphi\right)\right] \times 100 \%$, where $\Delta H_{\mathrm{m}}$ is the melting enthalpy, $\Delta H_{0}$ is a reference value (136 $\left.\mathrm{J} \mathrm{g}^{-1}\right)^{28}$ that represents the melting enthalpy of a pure PCL crystal, and $\varphi$ is the mass fraction of PCL in the composites. Thermal gravimetric analysis (TGA, TG209F3, Netzsch) was conducted from 30 to $800{ }^{\circ} \mathrm{C}$ under a $\mathrm{N}_{2}$ atmosphere at a heating rate of $10{ }^{\circ} \mathrm{C} \mathrm{min}^{-1}$. The mechanical properties were measured on a micro-controlled electronic universal testing machine (ETM502B-Ex, Wance Technologies Ltd). Tensile test procedures followed the ISO 1184-1983 method. The gas barrier properties of samples were measured using an oxygen permeability tester (OXTRAN 2/21, Mocon). Film specimens were cut into a circular shape with an area of $5 \mathrm{~cm}^{3}$. The measurement environment was set at $23 \pm 0.5^{\circ} \mathrm{C}$ with $0.1 \mathrm{MPa}$ oxygen pressure.

The minimum inhibitory concentration (MIC) method was carried out as follows. MIC values of Ag-LDHs were determined from the lowest concentration of Ag-LDHs that completely inhibited the growth of bacteria, i.e., there was no visible formation of colonies as judged by the naked eye. The Gram negative bacteria strain Escherichia coli $(E$. coli) was first cultured for $12 \mathrm{~h}$ in a fluid nutrient medium. And then an appropriate bacterial suspension was diluted until the absorbance value was about 0.1 at a wavelength of $600 \mathrm{~nm}$. After mixing $100 \mu \mathrm{L}$ of bacteria suspension and $900 \mu \mathrm{L}$ of fluid nutrient medium, Ag-LDHs powder with different concentrations ranging from 10 to $1000 \mu \mathrm{g} \mathrm{mL} \mathrm{m}^{-1}$ was dispersed uniformly in the bacteria suspensions and fluid nutrient medium. Then, the bacteria mixing liquid was placed in a constant temperature bath $\left(37^{\circ} \mathrm{C}\right)$ shaking bed for $2 \mathrm{~h}$. Afterwards, the bacteria mixing liquid $(0.1 \mathrm{~mL})$ was coated evenly on the solid nutrient medium and incubated for $18 \mathrm{~h}$ at $37^{\circ} \mathrm{C}$. Finally, the MIC values of AgLDHs were determined using the solid nutrient medium without bacterial growth.

The antibacterial properties of the Ag-LDHs/PCL nanocomposites were evaluated against $E$. coli using the inhibition zones method. Before testing, the $E$. coli strain was first cultured in a flask, and then the prepared $E$. coli solution was pipetted onto a plate and spread over the surface. A circular disk of each membrane $(d=10 \mathrm{~mm})$ was placed on the bacterial surface and incubated for $16 \mathrm{~h}$ at $37^{\circ} \mathrm{C}$. After that, the inhibition zones that formed around the films could serve as an indicator of the 
antibacterial properties and these were visually observed with a digital camera.

\section{Results and discussion}

\subsection{Structure of Ag-LDHs}

Three kinds of Ag-LDHs were prepared via the reduction of a silver precursor, $\left[\mathrm{Ag}\left(\mathrm{NH}_{3}\right)_{2}\right]^{+}$ions, with PVP, PDA and TA-Fe(III), respectively. TEM studies were carried out to investigate the morphology of AgNPs on the surface of the LDHs directly. After the reduction process, AgNPs are uniformly adsorbed on the surface of original LDHs, LDHs@PDA and LDHs@TA-Fe(III), leading to the formation of Ag-LDHs, as shown in Fig. 1. The average diameters of AgNPs on the LDHs can also be estimated as $\sim 25,65$ and $15 \mathrm{~nm}$, respectively, and, as shown in Fig. 2(a-c), AgNPs loading amounts on the LDHs are 3.37, 1.53 and $0.66 \mathrm{wt} \%$, respectively. All Ag-LDHs samples possess Ag(111) crystal planes with a lattice spacing of $0.236 \mathrm{~nm}$, as shown in the Fig. $2(\mathrm{~d}-\mathrm{f}){ }^{1}$ The successful preparation of AgNPs is further confirmed via XRD and UV-vis studies, as shown in Fig. 3. In Fig. 3(a), it can be seen that the diffraction reflections of LDHs are sharp and symmetrical, and the baselines are low and stable, indicating relatively well-formed crystalline layered structures. The (003), (006) and (110) characteristic diffraction peaks are easily recognized in all the patterns. XRD patterns of original LDHs and AgLDHs show almost identical characteristics to $\mathrm{MgAl}-\mathrm{CO}_{3}$ LDHs (JCPDS file no. 38-0487). ${ }^{29}$ In addition to the characteristic diffraction peaks of the original LDHs, (111), (200), and (220) diffraction peaks of $\mathrm{Ag}$ are observed in the XRD patterns of AgLDHs. ${ }^{30}$ This is consistent with the results from HRTEM images. As shown in Fig. 3(b), the original LDHs display no visible absorption peak between 300 and $800 \mathrm{~nm}$. After loading AgNPs on the surface of the LDHs, there are strong absorption bands at $\sim 420$ and $444 \mathrm{~nm} .{ }^{31}$ This is characteristic of spherical AgNPs, due to surface plasmon resonance (SPR) ${ }^{24}$ which further implies the formation of Ag-LDHs. The shapes, sizes and concentrations of nanoparticles, their morphologies and the reaction environments significantly affect the location and intensity of the SPR band. Compared with Ag-LDHs(PVP), Ag-LDHs@TA-Fe(III) shows a blue shift (from $444 \mathrm{~nm}$ to $420 \mathrm{~nm}$ ), which strongly reveals an abrupt decrease in the dimensional size of AgNPs. Although AgNPs of LDHs@PDA have a larger size than those of AgLDHs(PVP), the concentration of AgNPs in Ag-LDHs@PDA is lower than that of AgNPs in Ag-LDHs(PVP). Compared with Ag-
LDHs(PVP), Ag-LDHs@PDA still shows the blue shifting of the SPR band. In other words, a lower concentration of AgNPs may also lead to a blue shift in the SPR band. ${ }^{17}$

The photographs in Fig. 4 demonstrate the dispersibilities of LDHs and Ag-LDHs in aqueous solution. Original LDHs and three kinds of Ag-LDHs are respectively dispersed in ultrapure water via ultrasonication, and then the obtained dispersions are left to stand. After standing for $40 \mathrm{~min}$, LDHs and AgLDHs(PVP) obviously aggregate and precipitate, while AgLDHs@PDA and Ag-LDHs@TA-Fe(III) stay homogenously dispersed. After standing for $120 \mathrm{~min}$, LDHs and Ag-LDHs(PVP) completely precipitate, while Ag-LDHs@PDA and AgLDHs@TA-Fe(III) stay uniformly dispersed. The rather good dispersibilities of Ag-LDHs@PDA and Ag-LDHs@TA-Fe(III) can be attributed to the decreased surface energies of PDA and TA$\mathrm{Fe}(\mathrm{III})$, which are wrapped on the surface of the original LDHs; ${ }^{16}$ this can further improve the dispersibilities of Ag-LDHs@PDA and Ag-LDHs@TA-Fe(III) in the polymer matrix and correspondingly their performances.

\subsection{Antibacterial properties of Ag-LDHs and Ag-LDHs/PCL nanocomposites}

The minimum inhibitory concentrations (MICs) that would completely inhibit any visible growth of bacteria after overnight incubation have been quantified for Ag-LDHs. The differences in MIC values obtained are shown in Fig. 5 . There is a general consensus that agrees that $\mathrm{Ag}$ ions released from unstable AgNPs are responsible for its excellent antibacterial activity. High antibacterial activity performance is obtained if the AgNPs have a large surface area. A larger surface area contributes to the release of more $\mathrm{Ag}$ ions; as a result, more are exposed to bacteria. Therefore, the order of antimicrobial activity was correlated to the quantity of silver loading and the size of AgNPs. The test results can be directly correlated with the loading of AgNPs confirmed via EDS results and the sizes of AgNPs confirmed by TEM. The dependence of the antibacterial activity on these parameters has been previously reported. ${ }^{17}$ The medium size of the AgNPs present in Ag-LDHs(PVP) and the fact that it has the highest loading lead to it showing the best antibacterial properties. And the small size of the AgNPs present in Ag-LDHs@TA-Fe(III) and the fact that it has the lowest loading lead to it having the second-best antibacterial properties. Naturally, the large size of the AgNPs and the medium loading present in Ag-LDHs@PDA lead to it having the worst
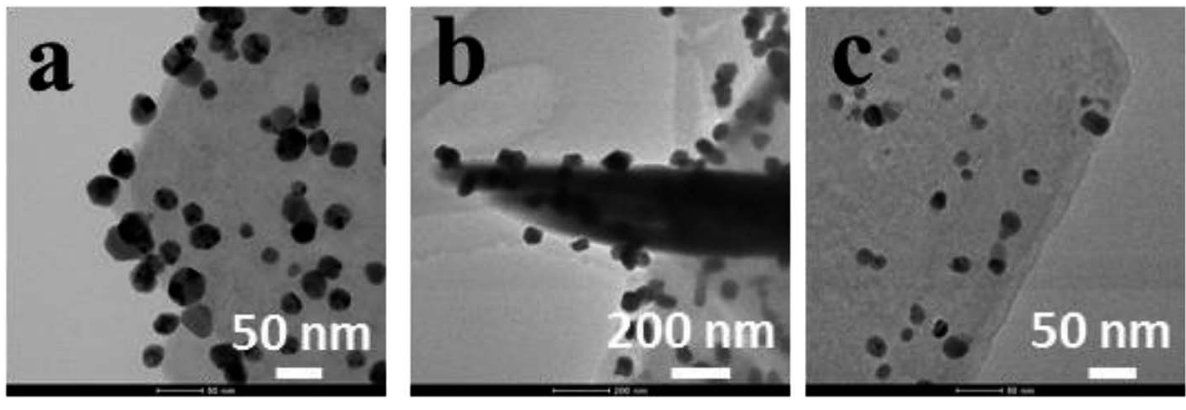

Fig. 1 TEM images of (a) Ag-LDHs(PVP), (b) Ag-LDHs@PDA and (c) Ag-LDHs@TA-Fe(III). 

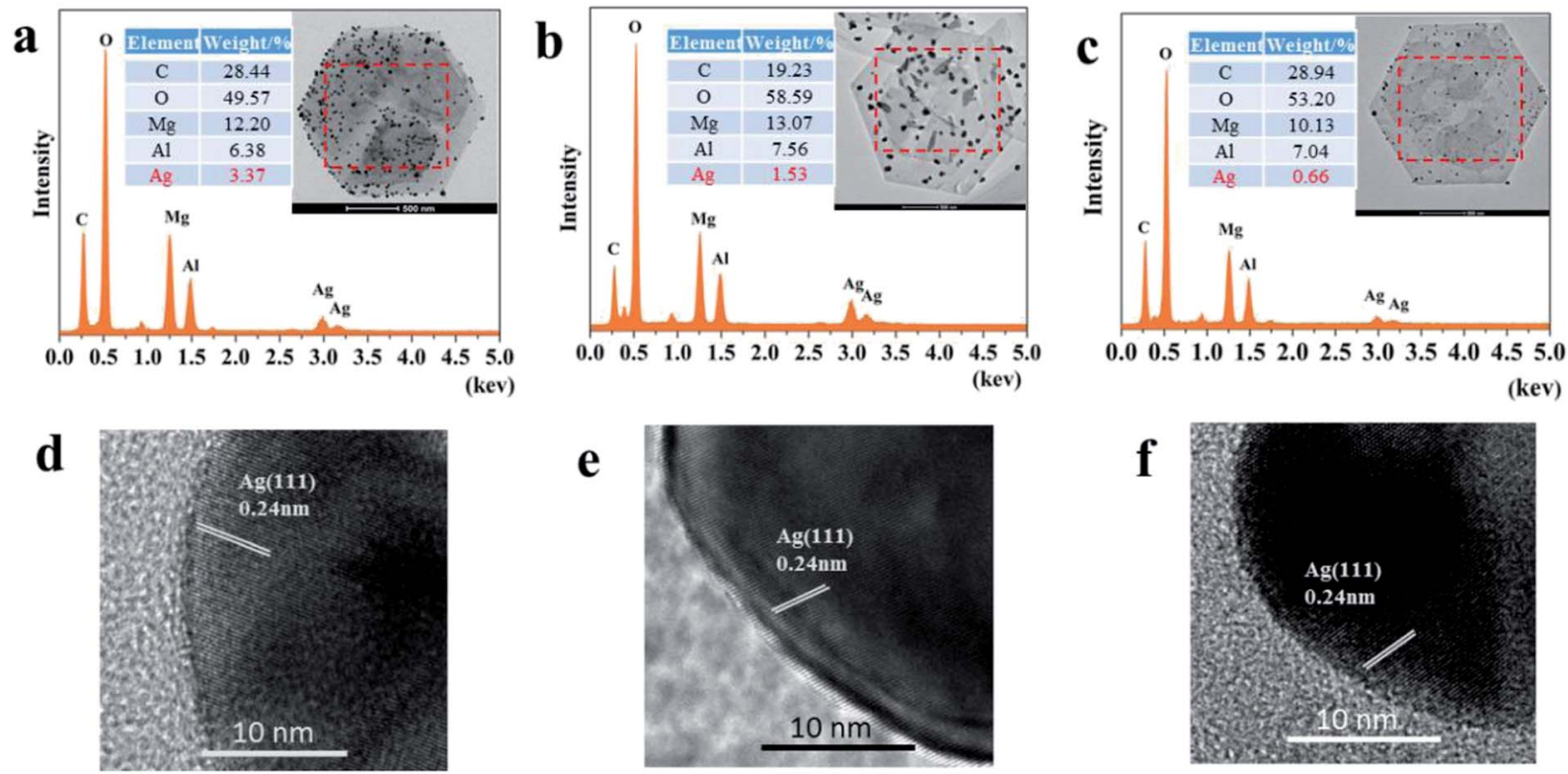

Fig. 2 EDS surface scans for (a) Ag-LDHs(PVP), (b) Ag-LDHs@PDA and (c) Ag-LDHs@TA-Fe(III). HRTEM images of (d) Ag-LDHs(PVP), (e) AgLDHs@PDA and (f) Ag-LDHs@TA-Fe(III).
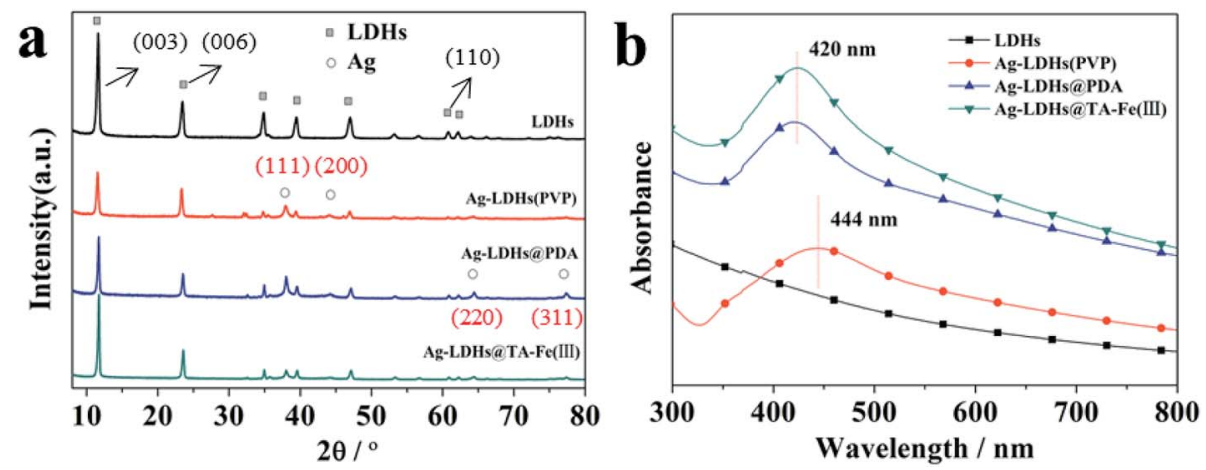

Fig. 3 (a) XRD patterns and (b) UV-vis spectra of LDHs and Ag-LDHs.

antibacterial properties. According to reports in the literature, ${ }^{30,32} \mathrm{Ag}$ ions released from the AgNPs contribute to the antibacterial activity. Therefore, LDHs with smaller sized AgNPs embedded in them and higher quantities of AgNP loading exhibit better antibacterial efficacy. Among the three kinds of Ag-LDHs, Ag-LDHs(PVP) exhibits the best antibacterial activity, in terms of having the lowest MIC value.

The inhibition zones of the Ag-LDHs/PCL nanocomposites against $E$. coli are shown in Fig. 6. Against $E$. coli, the inhibition zones of Ag-LDHs are observed to be 16.3, 14.1 and $15.9 \mathrm{~mm}$, respectively. With an increase in inhibition zone size, materials exhibit enhanced antibacterial activity. The Ag-LDHs(PVP)/PCL nanocomposite exhibits the best antibacterial activity. Obviously, the antibacterial activity of the Ag-LDHs/PCL nanocomposite is consistent with that of Ag-LDHs.

\subsection{Thermal properties of Ag-LDHs/PCL nanocomposites}

DSC cooling and secondary heating curves from Ag-LDHs/PCL nanocomposites are shown in Fig. 7, and the related results from thermal analysis are listed in Table 1. As seen from Fig. 7(a) and Table 1, pure PCL exhibits a crystallization exothermic peak $\left(T_{\mathrm{c}}\right)$ at $23.8{ }^{\circ} \mathrm{C}$, which is ascribed to the $\alpha$ polymorph.$^{33}$ With the addition of Ag-LDHs, the $T_{\mathrm{c}}$ and $\chi$ values of the Ag-LDHs/PCL nanocomposites occur at higher values. Therefore, increases in $T_{\mathrm{c}}$ and $\chi$ are due to the fact that all AgLDHs can act as heterogeneous nucleating agents to promote the crystallization process. ${ }^{34}$ In Fig. $7($ b), the melting temperature ( $\left.T_{\mathrm{m}}\right)$ of pure PCL is $57.3^{\circ} \mathrm{C}$ while $T_{\mathrm{m}}$ values of the Ag-LDHs/ PCL nanocomposites show no obvious changes $\left(56.5-57.1^{\circ} \mathrm{C}\right)$. This shows that Ag-LDHs samples do not obviously affect $T_{\mathrm{m}}$.

In order to study the relationship between LDH content and the crystallinities of the nanocomposites, Ag-LDHs@TA-Fe(III) is taken as an example to study the crystalline characteristics of Ag-LDHs/PCL nanocomposites (as shown in Fig. 8(a)). To a certain extent, the XRD patterns of Ag-LDHs@TA-Fe(III)/PCL nanocomposites further verify the results from DSC analysis. The diffraction intensities from LDHs and PCL in the nanocomposites increase simultaneously with the increase in Ag- 


\section{After 40 min}
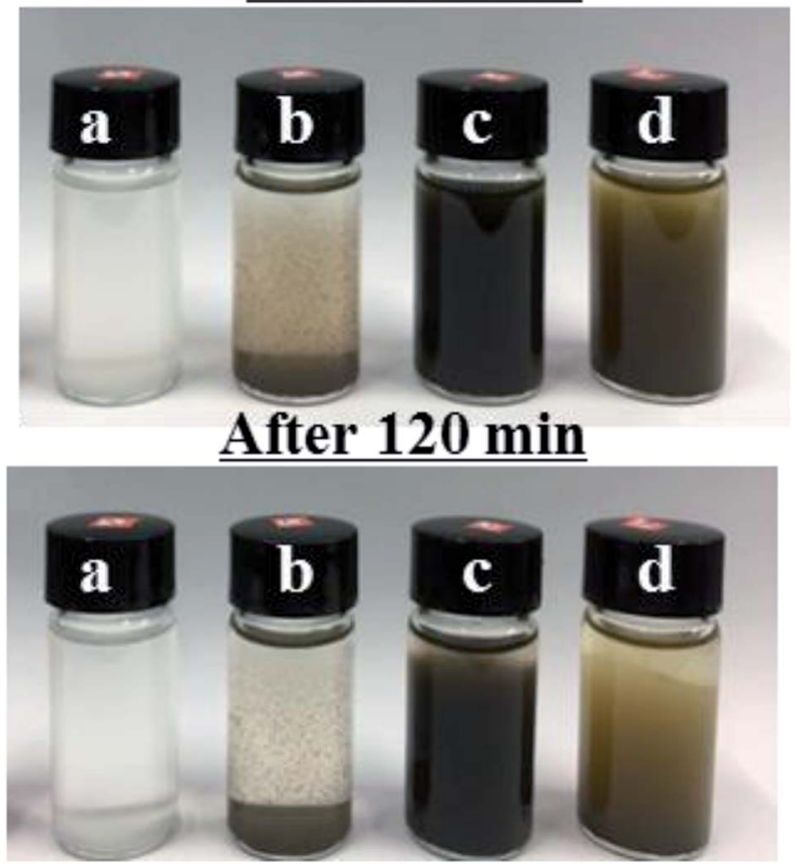

Fig. 4 Photographs of (a) LDHs, (b) Ag-LDHs(PVP), (c) Ag-LDHs@PDA and (d) Ag-LDHs@TA-Fe(III) dispersions after standing for $120 \mathrm{~min}$.

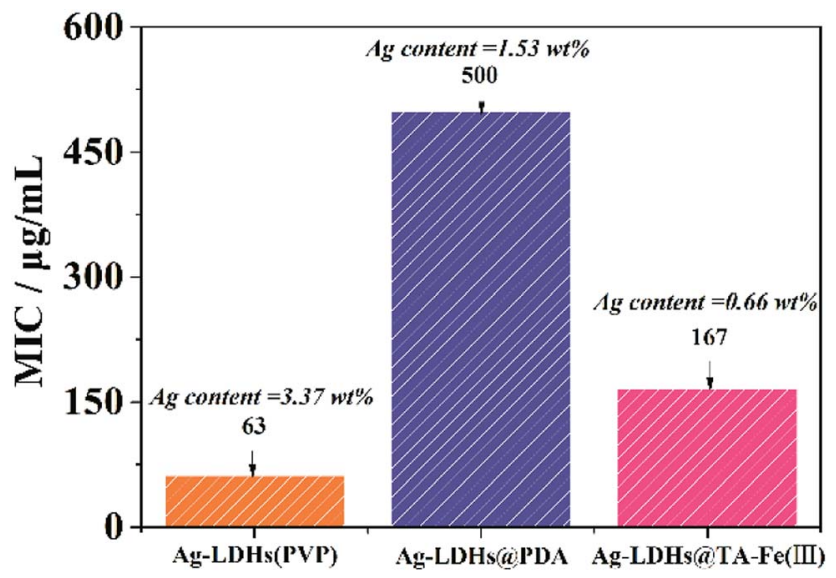

Fig. 5 The MIC values of Ag-LDHs.

LDHs@TA-Fe(III) amount. This reveals that Ag-LDHs@TA-Fe(III) facilitates the crystallization of PCL molecular chains as a result of an increase in crystallinity. It should be pointed out that the Ag-LDHs(PVP)/PCL and Ag-LDHs@PDA/PCL nanocomposites show the same trend. Therefore, Ag-LDHs can promote the crystallization process in the PCL matrix.

Fig. 8(b) shows the TGA curves from Ag-LDHs/PCL nanocomposites. According to previous studies, ${ }^{33,35}$ the thermal degradation of LDHs/PCL nanocomposites occurs at a lower temperature in comparison with pure PCL, and the thermal degradation temperature of LDHs/PCL nanocomposites decreases gradually as the $\mathrm{LDH}$ loading increases. It is revealed that the thermal degradation of the Ag-LDHs(PVP)/PCL and Ag-
LDHs@TA-Fe(III)/PCL nanocomposites shows the same trend. Interestingly, however, this is not the case for the AgLDHs@PDA/PCL nanocomposite. The Ag-LDHs@PDA/PCL nanocomposite exhibits better thermostability than pure PCL and the other Ag-LDHs/PCL nanocomposites. Owing to the high relative thermal stability of PDA, the core-shell structured AgLDHs@PDA contributes to a delay of the initial thermal degradation of PCL. ${ }^{6}$ Therefore, Ag-LDHs@PDA improves the thermostability of the Ag-LDHs@PDA/PCL nanocomposite in comparison to the other Ag-LDHs/PCL nanocomposites.

\subsection{Mechanical properties of Ag-LDHs/PCL nanocomposites}

The mechanical properties of materials are an important factor that can decide the usability, durability and reliability of manufactured goods. Fig. 9 shows the mechanical properties of the Ag-LDHs/PCL nanocomposites. In Fig. 9(a), the tensile strength of all the Ag-LDHs/PCL nanocomposites shows a similar trend; that is, the tensile strength decreases with an increase in AgLDHs content. When the addition of $\mathrm{Ag}-\mathrm{LDHs}$ is only $0.5 \mathrm{wt} \%$, compared with pure PCL (34.4 MPa), the tensile strengths of AgLDHs@PDA/PCL and Ag-LDHs@TA-Fe(III)/PCL nanocomposites decrease by $4 \%$ and $11 \%$, respectively, while the tensile strength of the Ag-LDHs(PVP)/PCL nanocomposite decreases by up to $26 \%$. There are some differences in the elongation at break curves for all the Ag-LDHs/PCL nanocomposites (Fig. 9(b)). With an increase in Ag-LDHs content, the elongation at break for all the Ag-LDHs/PCL nanocomposites first increases and then decreases quickly. When the addition of $\mathrm{Ag}-\mathrm{LDH}$ is $0.5 \mathrm{wt} \%$, the elongation at break for all the Ag-LDHs/ PCL nanocomposites reaches a maximum value. However, compared with pure PCL (612\%), the elongation at break values for the Ag-LDHs@PDA/PCL and Ag-LDHs@TA-Fe(III)/PCL nanocomposites increase by $50 \%$ and $39 \%$, respectively, and the elongation at break of the Ag-LDHs(PVP)/PCL nanocomposite increases by only $25 \%$. Therefore, compared with unmodified LDHs, the surface modification of LDHs has a remarkably positive effect on the mechanical properties of the PCL matrix.

To further understand the relationship between the material structure and mechanical properties, SEM images of the fractured surfaces of Ag-LDHs/PCL nanocomposites are shown in Fig. 10. In addition to a large number of bulges and hollows that can be observed in the fracture region (Fig. 10(a-c)), there are also numerous curled broken fibrils formed by plastic tension deformation, which can explain how the Ag-LDHs@PDA/PCL and Ag-LDHs@TA-Fe(III)/PCL nanocomposites exhibit larger elongation at break values. In Fig. $10(\mathrm{~d}-\mathrm{f})$, there is a clear gap between Ag-LDHs(PVP) and the PCL matrix, which indicates that the interfacial compatibility is relatively poor, however, AgLDHs@PDA and Ag-LDHs@TA-Fe(III) are both tightly combined with the PCL matrix, and there is no visible interface gap. This indicates that the interfacial compatibility is improved markedly, which can explain how the Ag-LDHs@PDA/PCL and AgLDHs@TA-Fe(III)/PCL nanocomposites exhibit better mechanical properties than the Ag-LDHs(PVP)/PCL nanocomposite. ${ }^{\mathbf{6}, 16}$ Moreover, elements of suspected AgNPs on the surfaces of the 


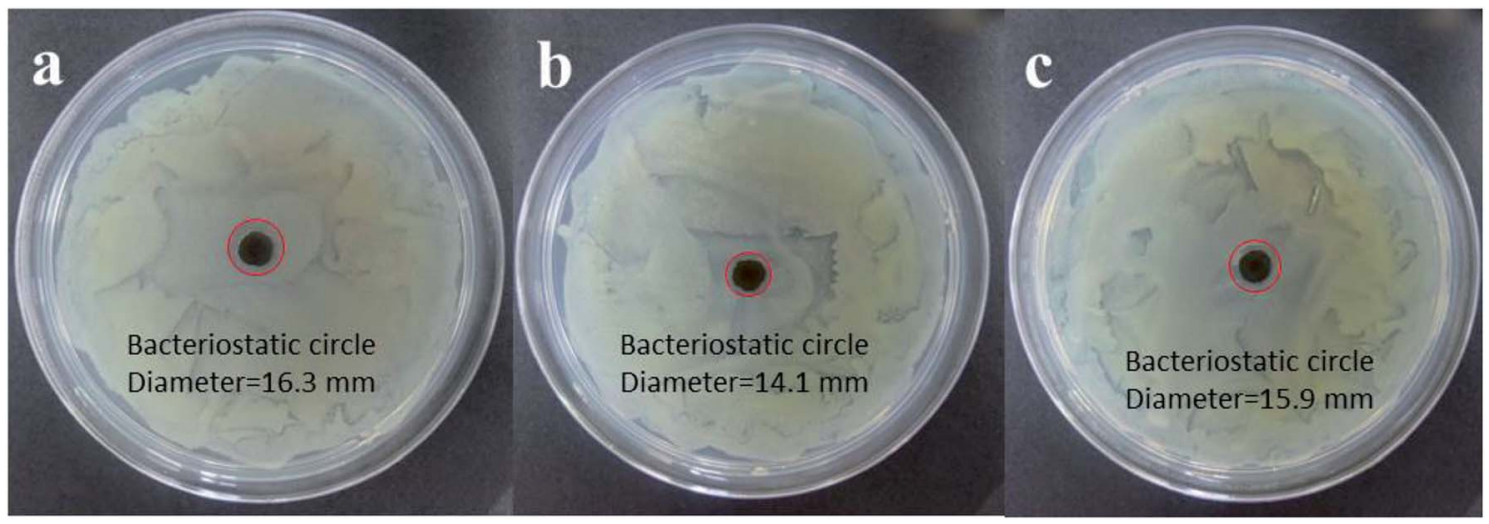

Fig. 6 The inhibition zone sizes against E. coli on different membranes: the (a) Ag-LDHs(PVP)/PCL nanocomposite; (b) Ag-LDHs@PDA/PCL nanocomposite; and (c) Ag-LDHs(aTA-Fe(III)/PCL nanocomposite.
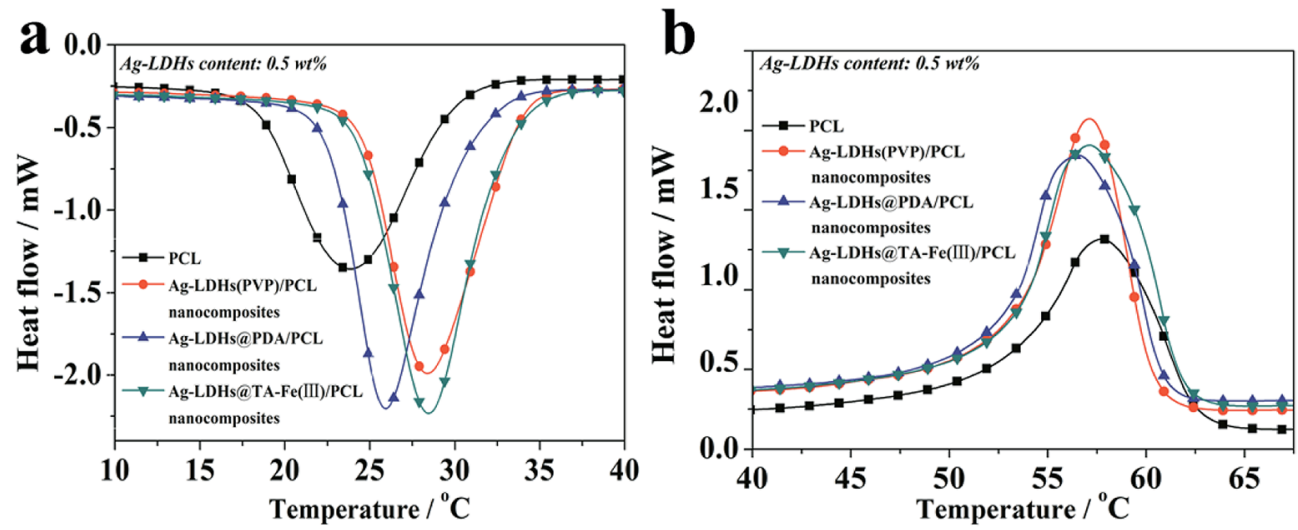

Fig. 7 DSC (a) cooling and (b) secondary heating curves of Ag-LDHs/PCL nanocomposites.

Table 1 The results for Ag-LDHs/PCL nanocomposites according to thermal analysis

\begin{tabular}{lllr}
\hline Sample & $\Delta H_{\mathrm{m}} / \mathrm{J} \mathrm{g}^{-1}$ & $T_{\mathrm{m}} /{ }^{\circ} \mathrm{C}$ & $T_{\mathrm{c}}{ }^{\circ} \mathrm{C}$ \\
\hline PCL & 45.18 & 57.3 & 23.8 \\
Ag-LDHs(PVP)/PCL nanocomposite & 52.70 & 57.1 & 33.2 \\
Ag-LDHs@PDA/PCL nanocomposite & 52.31 & 56.5 & 38.9 \\
Ag-LDHs@TA-Fe(III)/PCL nanocomposite & 57.94 & 57.1 & 38.7 \\
\end{tabular}
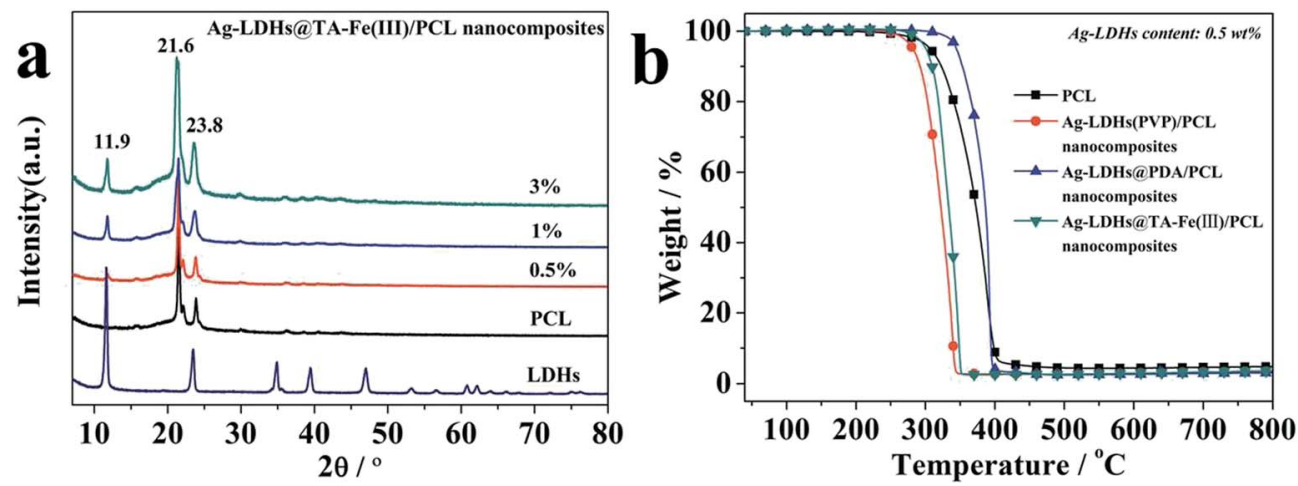

Fig. 8 (a) XRD patterns and (b) TGA curves of Ag-LDHs/PCL nanocomposites. 

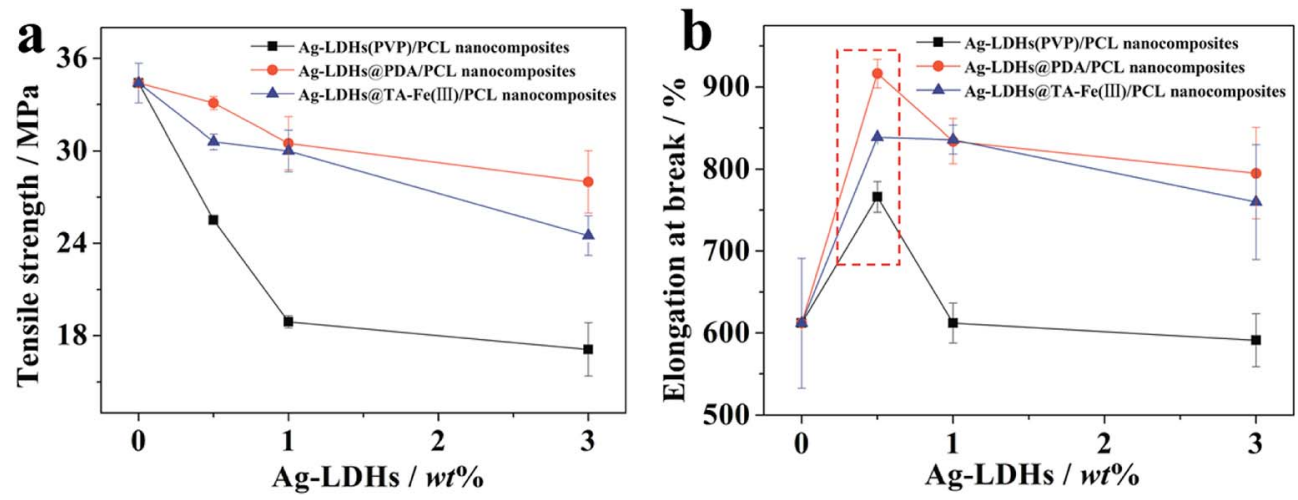

Fig. 9 The (a) tensile strength and (b) elongation at break curves for Ag-LDHs/PCL nanocomposites.
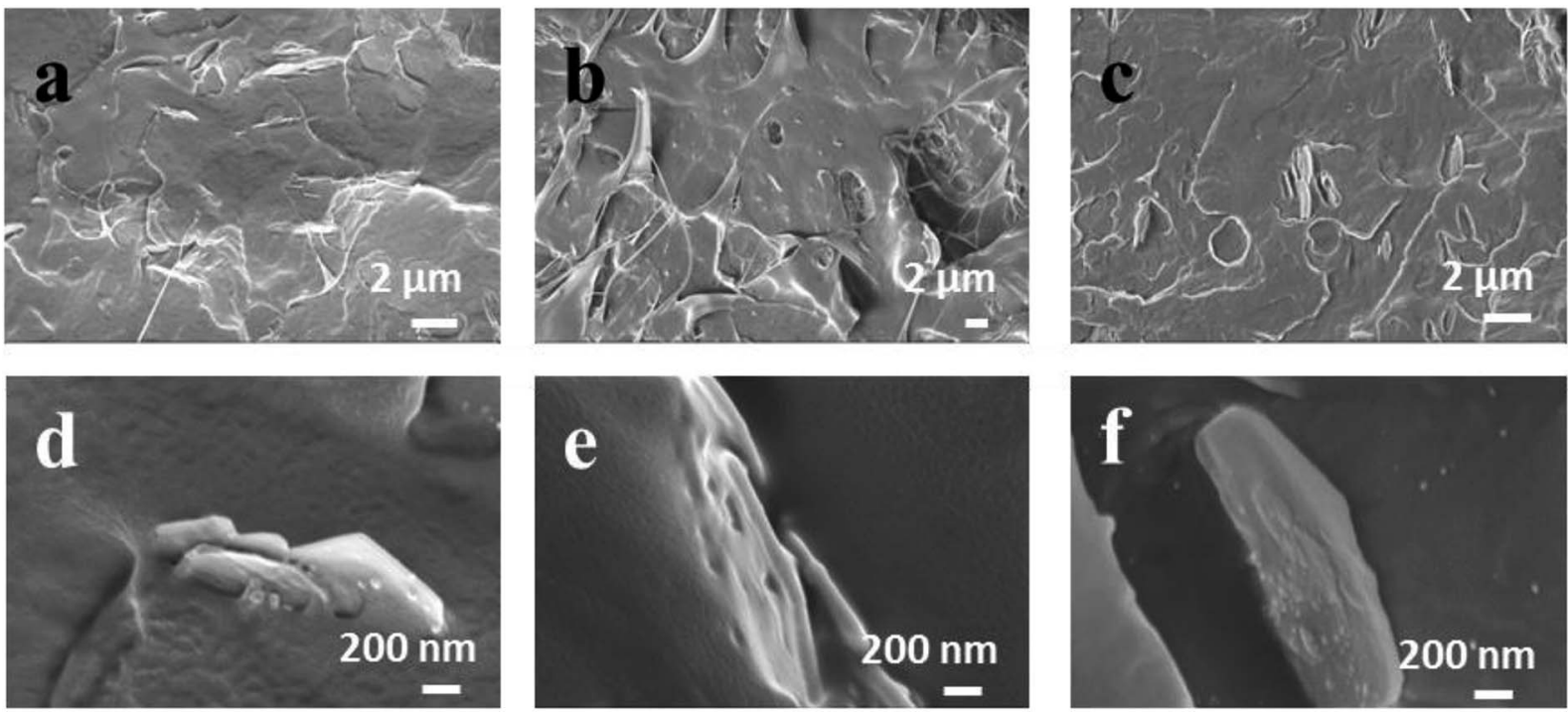

Fig. 10 SEM images of the fractured surfaces of Ag-LDHs/PCL nanocomposites at different magnifications (the Ag-LDHs content is 0.5 wt\%): (a and d) Ag-LDHs(PVP)/PCL nanocomposite; (b and e) Ag-LDHs(aPDA/PCL nanocomposite; and (c and f) Ag-LDHs@TA-Fe(III)/PCL nanocomposite.

LDHs are analyzed via EDS and the results show that Ag appears in the EDS spectra, which confirms that these spherical particles are AgNPs.

\subsection{Barrier properties of Ag-LDHs/PCL nanocomposites}

The oxygen permeability of the Ag-LDHs/PCL nanocomposites is shown in Fig. 11. With an increase in Ag-LDHs content, the oxygen permeabilities of all the Ag-LDHs/PCL nanocomposites decrease gradually. With the same amount of Ag-LDHs, the oxygen permeabilities of the Ag-LDHs/PCL nanocomposites show different values, which is related to the dispersion of AgLDHs itself in the PCL matrix and the interactions between $\mathrm{Ag}$-LDHs and the PCL matrices. In particular, when the addition of Ag-LDHs reaches $3 \mathrm{wt} \%$, the oxygen permeabilities of the $\mathrm{Ag}$ LDHs@PDA/PCL and Ag-LDHs@TA-Fe(III)/PCL nanocomposites decrease by $27 \%$ and $26 \%$, respectively. And their reduction values reach twice as much as that of the AgLDHs(PVP)/PCL nanocomposite. Combined with previous analysis, it is revealed that Ag-LDHs@PDA and Ag-LDHs@TA-

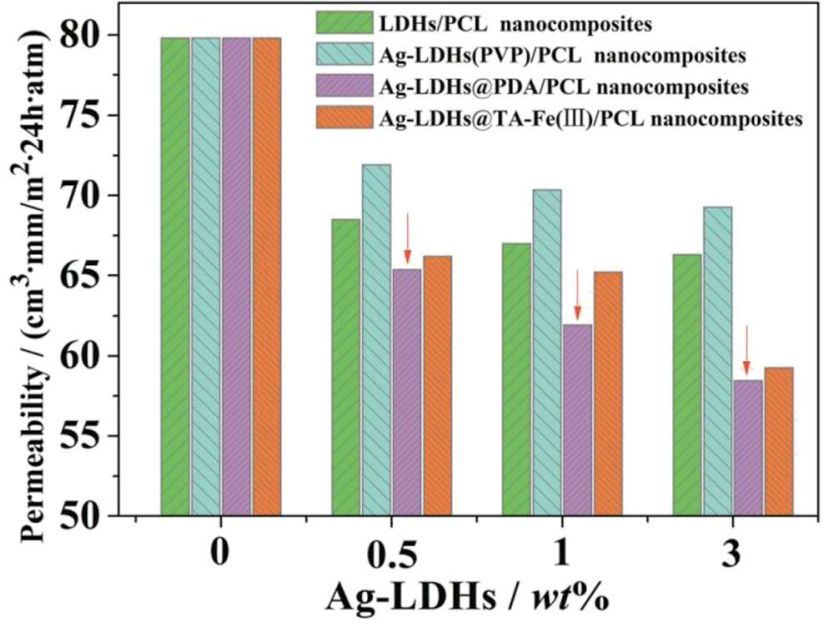

Fig. 11 The $\mathrm{O}_{2}$ permeability of the Ag-LDHs/PCL nanocomposites. 
$\mathrm{Fe}(\mathrm{III})$ are superior to $\mathrm{Ag}$-LDHs(PVP) in dispersion and interface interactions. The better dispersibilities of Ag-LDHs@PDA and Ag-LDHs@TA-Fe(III) contribute to the greater aspect ratios of $\mathrm{Ag}$-LDHs in the matrices, resulting in an increase in the number of tortuous paths for gas diffusion. At the same time, AgLDHs@PDA and Ag-LDHs@TA-Fe(III) have stronger interactions with the PCL matrix, which is favorable for the presence of less interface defects in the nanocomposites as a result of a decrease in the number density of free volume holes. ${ }^{36-38}$ Therefore, the Ag-LDHs@PDA/PCL and Ag-LDHs@TA-Fe(III)/ PCL nanocomposites show better oxygen barrier properties.

\section{Conclusions}

In this paper, a facile, green and mussel-inspired method was presented to prepare Ag-LDHs @PDA and Ag-LDHs@TA-Fe(III) using a pre-synthesis PDA/TA-Fe(III) layer as a nanoscale guide and PDA/TA itself as a reducing reagent to form uniform AgNPs on the surface of modified LDHs. Meanwhile, another kind of $\mathrm{Ag}$-LDHs(PVP) material was prepared via the direct reduction of silver precursor $\left[\mathrm{Ag}\left(\mathrm{NH}_{3}\right)_{2}\right]^{+}$ions with PVP. And three kinds of $\mathrm{Ag}$-LDHs/PCL nanocomposite were prepared by blending AgLDHs and pure PCL via a solution casting method to obtain homogeneous films. It is shown that the obtained AgNPs are distributed on the LDH surface uniformly. And the high loading and medium size of the AgNPs present in Ag-LDHs(PVP) result in it showing the best antibacterial properties. Increases in crystallization temperature and crystallinity with the incorporation of Ag-LDHs into the PCL matrix is due to the fact that all the Ag-LDHs materials can act as nucleating agents with resulting heterogeneous nucleation effects. Considering the high relative thermal stability of PDA, the core-shell structured Ag-LDHs@PDA contributes to a delay in the initial thermal degradation of PCL. Compared with Ag-LDHs(PVP), the better dispersibilities of Ag-LDHs@PDA and Ag-LDHs@TA-Fe(III) contribute to the greater aspect ratios of $\mathrm{Ag}-\mathrm{LDHs}$ in the matrices, resulting in an increase in the number of tortuous paths for gas diffusion. At the same time, Ag-LDHs@PDA and Ag-LDHs@TA-Fe(III) have stronger interactions with the PCL matrix, which is favorable for creating less interface defects in the matrix, resulting in increases in mechanical and gas barrier properties. Therefore, Ag-LDHs@PDA/PCL and Ag-LDHs@TAFe(III)/PCL nanocomposites show preferable mechanical and gas barrier properties. Thanks to the superiority of their physical properties, mussel-inspired antibacterial Ag-LDHs/PCL nanocomposites are promising materials that could be widely applied in the packaging and biomedical industries.

\section{Conflicts of interest}

The authors declare no competing financial interests.

\section{Acknowledgements}

This study was supported by the National Natural Science Foundation of China (No. 11872179 and 21704085), Science and Technology Planning Project of Fujian Province (No.
2018H6024), Young and Middle-aged Teachers Education Scientific Research Project of Fujian Province (No. JT180420 and JAT160354), Open fund of Fujian Provincial Key Laboratory of Functional Materials and Applications (Xiamen University of Technology) (No. fma2018004 and fma2017110), and Fujian Province Natural Science Fund (No. 2016J01322), Fujian Province Education Department Fund (No. JAT160351).

\section{References}

1 S. Zhao and J. Li, Silver-Cobalt Oxides Derived from Silver Nanoparticles Deposited on Layered Double Hydroxides for Methane Combustion, ChemCatChem, 2015, 7(13), 19661974.

2 J. Leng, N. Kang, D. Wang, et al., Structure-Property Relationships of Nanocomposites Based on Polylactide and Layered Double Hydroxides - Comparison of MgAl and NiAl LDH as Nanofiller, Macromol. Chem. Phys., 2017, 218(20), 1700232.

3 N. G. B. Allou, P. Saikia, A. Borah, et al., Hybrid nanocomposites of layered double hydroxides: an update of their biological applications and future prospects, Colloid Polym. Sci., 2017, 295(5), 725-747.

4 C. Wang, H. Han, W. Jiang, et al., Immobilization of Thermostable Lipase QLM on Core-Shell Structured Polydopamine-Coated $\mathrm{Fe}_{3} \mathrm{O}_{4}$ Nanoparticles, Catalysts, 2017, 7(2), 49-60.

5 H. J. Nam, E. B. Park and D. Jung, Bioinspired polydopamine-layered double hydroxide nanocomposites: controlled synthesis and multifunctional performance, $R S C$ Adv., 2016, 6(30), 24952-24958.

6 L. Mao, Y. Liu, H. Wu, et al., Poly( $\varepsilon$-caprolactone) filled with polydopamine-coated high aspect ratio layered double hydroxide: simultaneous enhancement of mechanical and barrier properties, Appl. Clay Sci., 2017, 150, 202-209.

7 L. P. Si, L. Yang, S. Huang, et al., Shape memory polyurethane with polydopamine-coated nanosheets: simultaneous enhancement of recovery stress and strain recovery ratio and the underlying mechanisms, Eur. Polym. J., 2014, 57(8), 11-21.

8 H. J. Nam, E. B. Park and D. Jung, Bioinspired polydopamine-layered double hydroxide nanocomposites: controlled synthesis and multifunctional performance, $R S C$ Adv., 2016, 6(30), 24952-24958.

9 J. Xie, K. Zhang, J. Wu, et al., Bio-nanocomposite films reinforced with organo-modified layered double hydroxides: preparation, morphology and properties, Appl. Clay Sci., 2016, 126, 72-80.

10 T. Xu, J. Zhang, H. Chi, et al., Multifunctional properties of organic-inorganic hybrid nanocomposites based on chitosan derivatives and layered double hydroxides for ocular drug delivery, Acta Biomater., 2016, 36, 152-163.

11 Q. Song, W. Zhao, H. Yin, et al., Facile synthesis of FeIIItannic acid film-functionalized magnetic silica microspheres for the enrichment of low-abundance peptides and proteins for MALDI-TOF MS analysis, $R S C$ $A d v .$, 2015, 5(78), 63896-63902. 
12 H. Ejima, J. J. Richardson, K. Liang, et al., One-step assembly of coordination complexes for versatile film and particle engineering, Science, 2013, 341(6142), 154-157.

13 M. Božič, S. Gorgieva and V. Kokol, Homogeneous and heterogeneous methods for laccase-mediated functionalization of chitosan by tannic acid and quercetin, Carbohydr. Polym., 2012, 89(3), 854-864.

14 G. Kang, M. Liu, B. Lin, et al., A novel method of surface modification on thin-film composite reverse osmosis membrane by grafting poly(ethylene glycol), Polymer, 2007, 48(5), 1165-1170.

15 H. J. Kim, Y. Choi, M. Lim, et al., Reverse osmosis nanocomposite membranes containing graphene oxides coated by tannic acid with chlorine-tolerant and antimicrobial properties, J. Membr. Sci., 2016, 514, 25-34.

16 L. Mao, H. Wu, Y. Liu, et al., Enhanced mechanical and gas barrier properties of poly( $\varepsilon$-caprolactone) nanocomposites filled with tannic acid-Fe(III) functionalized high aspect ratio layered double hydroxides, Mater. Chem. Phys., 2018, 211, 501-509.

17 A. Roy, B. S. Butola and M. Joshi, Synthesis, characterization and antibacterial properties of novel nano-silver loaded acid activated montmorillonite, Appl. Clay Sci., 2017, 146, 278285.

18 F. B. Karel, A. S. Koparal and E. Kaynak, Development of Silver Ion Doped Antibacterial Clays and Investigation of Their Antibacterial Activity, Adv. Mater. Sci. Eng., 2015, 2015, 1-6.

$19 \mathrm{H}$. Wu, J. Huang and Y. Liu, Polysulfone ultrafiltration membrane incorporated with $\mathrm{Ag}_{-} \mathrm{SiO}_{2}$ nanohybrid for effective fouling control, J. Water Health, 2017, 15(3), 341352 .

20 M. Lavorgna, I. Attianese, G. G. Buonocore, et al., MMTsupported Ag nanoparticles for chitosan nanocomposites: structural properties and antibacterial activity, Carbohydr. Polym., 2014, 102(3), 385-392.

21 G. Cao, Y. Sun, J. Chen, et al., Sutures modified by silverloaded montmorillonite with antibacterial properties, Appl. Clay Sci., 2014, 93-94, 102-106.

22 A. L. Incoronato, G. G. Buonocore, A. Conte, et al., Active systems based on silver-montmorillonite nanoparticles embedded into bio-based polymer matrices for packaging applications, Journal of Food Protection, 2010, 73(12), 22562262.

23 Y. Cong, T. Xia, M. Zou, et al., Mussel-inspired polydopamine coating as a versatile platform for synthesizing polystyrene/Ag nanocomposite particles with enhanced antibacterial activities, J. Mater. Chem. B, 2014, 2(22), 3450-3461.

24 Z. Zhang, J. Zhang, B. Zhang, et al., Mussel-inspired functionalization of graphene for synthesizing Agpolydopamine-graphene nanosheets as antibacterial materials, Nanoscale, 2013, 5(1), 118-123.

25 R. Patwa, A. Kumar and V. Katiyar, Effect of silk nano-disc dispersion on mechanical, thermal, and barrier properties of poly(lactic acid) based bionanocomposites, J. Appl. Polym. Sci., 2018, 135(38), 46671.
26 A. Boujemaoui, C. Cobo Sanchez, J. Engström, et al., Polycaprolactone Nanocomposites Reinforced with Cellulose Nanocrystals Surface-Modified via Covalent Grafting or Physisorption: A Comparative Study, ACS Appl. Mater. Interfaces, 2017, 9(40), 35305-35318.

27 L. Mao, Y. Liu, Y. Bai, et al., Poly( $\varepsilon$-caprolactone) nanocomposites with layered double hydroxides modified by in situ grafting polymerization: structure characterization and barrier properties, J. Appl. Polym. Sci., 2017, 134(38), 45320-45326.

28 L. N. Ludueña, V. A. Alvarez and A. Vazquez, Processing and microstructure of PCL/clay nanocomposites, Mater. Sci. Eng., A, 2007, 460-461, 121-129.

29 Y. Liu, L. Mao and S. Fan, Preparation and study of intumescent flame retardant poly(butylene succinate) using MgAlZnFe- $\mathrm{CO}_{3}$ layered double hydroxide as a synergistic agent, J. Appl. Polym. Sci., 2014, 131(17), 89648973.

30 X. Wang, Z. Liang, F. Zhang, et al., Enhanced catalytic performances of $\mathrm{Ag}$ nanoparticles supported on layered double hydroxide for styrene epoxidation, J. Mater. Sci., 2013, 48(17), 5899-5903.

31 G. Xu, X. Qiao, X. Qiu, et al., Preparation and characterization of nano-silver loaded montmorillonite with strong antibacterial activity and slow release property, J. Mater. Sci. Technol., 2011, 27(8), 685-690.

32 S. C. Motshekga, S. S. Ray, M. S. Onyango, et al., Microwaveassisted synthesis, characterization and antibacterial activity of Ag/ZnO nanoparticles supported bentonite clay, J. Hazard. Mater., 2013, 262(8), 439-446.

33 R. Pucciariello, L. Tammaro, V. Villani, et al., New nanohybrids of poly( $\varepsilon$-caprolactone) and a modified $\mathrm{Mg} / \mathrm{Al}$ hydrotalcite: mechanical and thermal properties, J. Polym. Sci., Part B: Polym. Phys., 2007, 45(8), 945-954.

34 H. Peng, Y. Han, T. Liu, et al., Morphology and thermal degradation behavior of highly exfoliated CoAl-layered double hydroxide/polycaprolactone nanocomposites prepared by simple solution intercalation, Thermochim. Acta, 2010, 502(1-2), 1-7.

35 U. Costantino, V. Bugatti, G. Gorrasi, et al., New Polymeric Composites Based on Poly( $\varepsilon$-caprolactone) and Layered Double Hydroxides Containing Antimicrobial Species, ACS Appl. Mater. Interfaces, 2009, 1(3), 668-677.

36 R. K. Layek, A. K. Das, M. J. Park, et al., Enhancement of physical, mechanical, and gas barrier properties in noncovalently functionalized graphene oxide/ poly(vinylidene fluoride) composites, Carbon, 2015, 81(1), 329-338.

37 D. Aldana, E. Villa, M. De Dios Hernández, et al., Barrier properties of polylactic acid in cellulose based packages using montmorillonite as filler, Polymers, 2014, 6(9), 23862403.

38 S. Zekriardehani, S. A. Jabarin, D. R. Gidley, et al., Effect of chain dynamics, crystallinity, and free volume on the barrier properties of poly(ethylene terephthalate) biaxially oriented films, Macromolecules, 2017, 50(7), 2845-2855. 